\section{Cahiers de Narratologie}

Analyse et théorie narratives

8 | 1997

Création de l'espace et narration littéraire

\title{
L'espace dans le roman épistolaire : La serafina de José Mor De Fuentes (1797)
}

\section{Marc Marti}

\section{(2) OpenEdition}

1 Journals

Édition électronique

URL : http://journals.openedition.org/narratologie/11607

DOI : 10.4000/narratologie. 11607

ISSN : 1765-307X

Éditeur

LIRCES

\section{Édition imprimée}

Date de publication : 1 décembre 1997

Pagination : 263-276

ISBN : 291089746X

ISSN : 0993-8516

\section{Référence électronique}

Marc Marti, «L'espace dans le roman épistolaire : La serafina de José Mor De Fuentes (1797) », Cahiers de Narratologie [En ligne], 8| 1997, mis en ligne le 15 décembre 2020, consulté le 25 février 2021. URL: http://journals.openedition.org/narratologie/11607; DOI : https://doi.org/10.4000/narratologie.11607

Ce document a été généré automatiquement le 25 février 2021.

Article L.111-1 du Code de la propriété intellectuelle. 


\title{
L'espace dans le roman épistolaire : La serafina de José Mor De Fuentes (1797)
}

\author{
Marc Marti
}

\section{Introduction}

1 Le roman de José Mor de Fuentes, La Serafina, a été publié à Madrid en 1797. Il semble que le succès qu'il a rencontré explique les deux éditions postérieures réalisées respectivement en 1802 et 1807 - il y eut aussi deux éditions pirates en Barcelone en 1798. Pour le travail qui suit, nous utiliserons le texte de 1807, que reproduit Manuel Gil Ildefonso dans l'édition la plus récente à ce jour (Saragosse 1959) ${ }^{1}$.

2 L'argument du roman est simple. Le principal protagoniste, Alfonso, écrit la totalité des lettres qui relatent les progrès de son amour et ses fiançailles avec Serafina, une jeune Saragossaine. L'unique destinataire de la correspondance est Eugenio, ami d'Alfonso, qui réside à Burgos. Les péripéties se limitent à l'apparition de rivaux amoureux, quelques promenades champêtres et une attaque de brigands. Ce roman peut donc indéniablement être mis en relation avec la littérature européenne du XVIII ${ }^{\mathrm{e}}$ : d'abord bien sûr par sa forme épistolaire mais aussi par sa thématique et son ambiance qui sont celles du roman domestique, à savoir, un rythme lent, basé sur de petits événements de la vie quotidienne situés dans un monde familier².

3 C'est en ce sens que la création de l'espace dans ce roman nous intéresse. Quant à la méthodologie, nous distinguerons trois niveaux de lecture de l'espace, nous inspirant en cela d'un article récent de Jacques Soubeyroux ${ }^{3}$ :

- un premier niveau superficiel, qui correspond à une «topographie mimétique». Nous supposerons que le texte romanesque utilise des éléments qui dénotent la réalité pour construire son propre espace. Ce degré de mimétisme, plus ou moins accentué suivant les époques et les genres est particulièrement élevé dans le roman de Mor de Fuentes. L'analyse de ce niveau nécessite un relevé systématique des indicateurs spatiaux, c'est-à-dire les 
signes du texte à partir desquels se construit l'illusion de réalité spatiale dans le roman. Il sera cependant nécessaire de prendre en compte le fait que certaines lettres ne contiennent aucun indicateur spatial, construisant ainsi un espace implicite, qui n'a pas de relation directe avec la topographie.

- Le second niveau, celui de la «toposémie fonctionnelle» se situe au niveau du fonctionnement interne du texte. On considérera ici l'espace comme un véritable actant qui participe au développement du récit. Il conviendra par ailleurs d'analyser les différentes fonctions de l'espace ainsi que ses relations avec les personnages et les techniques narratives mises en œuvre dans le texte.

- Le troisième niveau, plus profond, correspond au "symbolisme idéologique ». Nous considérerons que l'espace romanesque diffère de la description objective, et que, au-delà de sa valeur fonctionnelle, il renvoie aussi à des représentations mentales plus ou moins conscientes qui sont régies par un code de valeurs - esthétiques, morales ou sociales -, la plupart du temps implicite.

\section{Un espace mimétique}

4 Le roman se construit à partir d'une double spatialité, ou du moins il convient de distinguer deux dimensions spatiales : l'espace épistolaire et l'espace topographique.

\subsection{L'espace épistolaire}

5 Généralement, tout roman épistolaire établit, implicitement ou explicitement, un pacte de lecture qui consiste en l'acceptation du fait que le texte est la transcription fidèle de lettres échangées entre différentes personnes. Dans le cas de La Serafina, le pacte se réduit aux éléments minimaux: l'ensemble des lettres a été écrit par Alfonso et l'unique destinataire en est Eugenio. Ce dernier révèle dans une postface ou note finale qu'il est l'éditeur de ces lettres.

6 Au bout du compte, la forme épistolaire comme tout type d'acte énonciatif, crée un espace qui n'est pas topographique mais communicatif. Un espace qui permet la mise en contact entre Alfonso et Eugenio et dont les uniques indices textuels sont les dates au bas des lettres - le lieu n'apparaissant que lorsque Alfonso séjourne en dehors de Zaragosse. Le lecteur, récepteur au second degré, fait lui aussi partie de cet espace. Nous pouvons identifier ce phénomène, selon les termes de Gérard Genette, comme une « spatialité littéraire active » ou « une spatialité représentative mais non représenté ${ }^{4}$ ». Cette spatialité est bien sûr présente dans tous les textes littéraires, mais une des particularités de la littérature épistolaire est de la mettre en scène.

7 Selon Laurent Versini, l'aspiration à l'authenticité et à la vraisemblance explique cet artifice $^{5}$. Dans le texte de La Serafina, l'illusion d'authenticité que procure la forme épistolaire est renforcée par l'accumulation de petits détails, parmi lesquels se trouvent les détails spatiaux, avec des références topographiques.

\subsection{L'espace topographique}

8 Nous considérerons comme des espaces topographiques tous les espaces construits à partir de signes se référant à la réalité. Dans le roman étudié, ce type d'espace se caractérise avant tout par une construction basée sur les toponymes, qui en majorité 
désignent la ville de Saragosse ou des lieux de cette même ville. Il s'agit de quartiers ou de places « la Cuchillería, la plaza del Pilar (lettres 11 y 16) »; de promenades « el Coso, Santa Engracia (lettre 14), Torrero (lettres 12, 53, 116)»; portes de la ville « Puerta de Sancho (lettre 5) puerta del Angel (lettre 111) " ou des abords immédiats de la ville « orilla del Ebro (lettres 5, 8, 94) ». D'autres espaces apparaissent, uniquement désignés par une appellation générique "rue (calle), ruelle (callejuela), vergers (huertas), promenades (paseos)». Complétant ce cadre urbain, nous avons, «le balcon de la maison de Serafina" (el balcón de la casa de Serafina) et un des principaux lieux du roman, "la maison de Serafina ", (la casa de Serafina) qui apparait implicitement ou explicitement dans une trentaine de lettres. De la même manière, sont mentionnées les maisons d'Alfonso ou celles des personnages secondaires (Rosalía, don Felix etc.).

En parallèle à ce décor urbain se trouvent des espaces ruraux, lieux de séjour du protagoniste : Daroca (lettres 72 a 76) et Villamayor (lettres 84 à 96), deux villages de la périphérie de Saragosse. La campagne apparaît aussi dans les lettres 127 et 131. C'est un espace de divertissement, évoqué par des descriptions schématiques.

Globalement, l'espace diégétique de La Serafina est essentiellement urbain et il convient de remarquer que le trait principal de cet espace - si l'on excepte l'emploi des toponymes - est la rareté des descriptions. Celles-ci consistent presque toujours en des visions panoramiques sur les alentours de Saragosse et en des évocations de lieux de promenade, c'est-à-dire uniquement des espaces-paysages. La ville au contraire ne bénéficie d'aucune description.

11 Il en va presque de même pour les espaces extra-diégétiques, qui apparaissent généralement dans des récits emboîtés, formés par les souvenirs d'Alfonso : « Madrid (lettre 1, 40,70); el Prado (lettre 1), Valence (lettre 51), Burgos » etc.

12 La fonction première de l'espace, construit à base de toponymes est, comme l'affirme Roland Barthes, de dénoter la réalité6. Mais l'espace littéraire ne peut être réduit à cet aspect, car il fonctionne aussi de façon métonymique dans le récit, assurant des fonctions narratives indéniables ${ }^{7}$.

\section{Toposémie fonctionnelle}

\subsection{Sociabilité, vraisemblance et structure du récit}

La plupart des lieux dans le roman de Mor de Fuentes pourraient être qualifiés d'espaces de sociabilité, c'est-à-dire des espaces dont la fonction est de favoriser la rencontre entre les différents personnages (rues, promenades, théâtre, novilladas, tertulias)

14 Il convient d'abord de remarquer que ces lieux répètent le postulat de sociabilité qui est le fondement même de l'espace de communication du genre épistolaire ${ }^{8}$. Ils servent aussi la vraisemblance, rendant possible, donc vraisemblable, la rencontre entre personnages. Mais au-delà de cette fonction, ces espaces de sociabilité se transforment aussi en espaces narratifs. La maison de Serafina, qui réunit toujours les mêmes personnes est un lieu fermé, espace du récit principal. Au contraire, les rues, les promenades, le théatre permettent le développement de récits secondaires comme le mariage malheureux de Narcisa, raconté grâce aux circonstances spatiales :

carta 43 : «Hier matin, je déambulais sur Santa Engracia, perdu dans mes pensées, lorsque je vis une dame assise, qui me regarda de façon insistante ; je m'en avisai et 
en m'approchant, je reconnus, non sans mal, Narcisa, qui était si affaiblie et défigurée que je n'arrivais pas à croire que ce fût elle.

A peine la saluai-je, que je lui demandai instamment qu'elle me fît part de ses malheurs, ainsi que de sa situation présente. Elle me parla en ces termes : [...]» différentes rencontres se déroulent dans la rue, soit lors des promenades, soit lorsque Serafina apparaît au balcon. Cet endroit est d'ailleurs ambivalent, véritable frontière entre l'espace privé, l'intimité de la maison et l'espace public de la rue; il disparaîtra définitivement après la lettre 41 , lorsque Alfonso aura accès librement à la maison de sa belle. De la même façon, la rue et les promenades vont perdre de l'importance en faveur de la maison, à partir du moment où Alfonso est admis à participer aux tertulias se déroulant chez Serafina. Cependant, le trajet spatial du protagoniste principal comporte deux déviations qui correspondent exactement à la détérioration de ses relations amoureuses. A l'éloignement sentimental correspond donc un éloignement spatial, loin de la ville. D'abord le séjour à Daroca, qui favorise l'apparition d'un rival (lettres 72 à 76), ensuite celui à Villamayor (lettres 84 à 96), qui constitue une sorte d'exil volontaire avec d'évidentes relations intertextuelles avec l'épisode de la Sierra Morena du Quichotte, auquel Alfonso fait explicitement référence : «Je suis revenu hier de mon expédition solitaire et quichottesque ${ }^{10} »$.

19 Schématiquement donc, l'espace se dessine en cercles concentriques dont les marges correspondent à la campagne, ensuite nous avons la ville avec ses rues et ses promenades et au centre l'intimité des tertulias dans la maison de Serafina, qualifiée de « sanctuaire » (lettre 56).

20 Ce dernier exemple nous oriente vers la notion de perception et de construction de l'espace, c'est-à-dire par quels moyens et de quelle façon est évoqué l'espace dans ce roman.

\subsection{La perception de l'espace}

21 Nous avons déjà démontré que l'espace se construit avant tout à travers les toponymes et les noms génériques, dans un souci de vraisemblance. Cependant, les quelques 
descriptions qui vont au delà de cette technique minimaliste, sont intéressantes à analyser. Prenons un exemple.

Lettre 85 : Ami, si j'étais astronome et n'étais pas amoureux, je serais au paradis avec cet observatoire.

Un ciel clair, telle est la voûte brillante de la perspective qui m'entoure. Au nord se détache majestueusement les Pyrénées d'où dévale le Gállego cristallin, qui vient arroser sur des lieues et des lieues cette terre fertile, composée en grande partie de vignes exubérantes. Au couchant, d'un côté, la vue se s'étire sur le courant paisible de l'Ebre et les plaines de la Bardena, jusqu'à se perdre au cœur de la Navarre.

Et de l'autre côté, le Moncayo doré

Brille, d'un éclair lumineux

De nuages crépusculaires

Qui sur son front reposent

car à cette époque, on ne voit pas « son front haut, de neige couronné » comme dit mon compatriote Argensola. Presque à midi, on distingue parfaitement Saragosse, située dans la plaine, avec ses hautes tours qui la rendent plus visible de loin que Valence, Barcelone et d'autre villes importantes. Derrière elle, on aperçoit l'ancien désert de Torrero, transformé en délicieux verger grâce à la sollicitude de l'immortel Pignatelli. Plus à gauche, l'Ebre réapparait et puis au levant il est dévié et rentre dans les gorges des montagnes les plus proches, presque toutes stériles et dont le triste aspect figure le clair-obscur de ce tableau incomparable ${ }^{11} »$.

Dans cette lettre, totalement consacrée à une description, différentes techniques narratives sont mises en œuvre. D'abord, le point de départ est un lieu qui rend vraisemblable la vision panoramique («observatoire»). Ensuite, il s'agit d'une description focalisée par Alfonso «Ami, si j'étais astronome [...] je serais au paradis avec cet observatoire ».

Ensuite, cet espace est construit en perspective ou profondeur, on passe en effet des éléments lointains aux éléments les plus proches. Il s'agit aussi d'un espace scrupuleusement délimité par les points cardinaux ( Au nord, au couchant, presqu'à midi, plus à gauche etc. ») et un horizon fermé par les Pyrénées.

Les objets qui constituent le paysage fonctionnent de façon dialectique, les éléments naturels sauvages («Pyrénées, montagnes») s'opposent avec ceux que l'homme a transformé (« jardins, vignes, vergers »).

Ces techniques de perception et de construction de l'espace renvoient aux idées esthétiques de l'époque. Le texte de Mor de Fuentes se situe à une période où le roman n'a pas encore acquis son autonomie descriptive. L'évocation d'espaces naturels se fait grâce à des techniques héritées de la poésie. On retrouve par exemple la fonction ornementale qu'avaient les descriptions. Le phénomène est ainsi un dérivé du locus amœnus gréco-latin revisité par l'esthétique rococo qui privilégie dans le texte la création d'éléments ornementaux autonomes, ce qui picturalement correspondrait à des miniatures ou de charmants tableautins ${ }^{12}$.

Cette esthétique se combine avec la vision de la nature propre aux Lumières, symétrie et construction géométrique ${ }^{13}$.

En parallèle à ce style disons poétique et illustré par l'exemple précédent, on remarque dans l'ensemble du roman l'émergence de techniques qui préfigurent le réalisme, en particulier l'abondance de lieux de sociabilité, espaces privilégiés de l'action. On peut avancer l'idée de la naissance d'une poétique urbaine romanesque : la ville et ses lieux de rencontre se transforme en un espace actif dans le déroulement narratif, un espace romanesque. Cependant, l'abondance de toponymes urbains, privés de toute 
description, contrairement à la campagne, nous amène à nous interroger sur la vision ville/campagne qui sous-tend l'espace romanesque.

\section{Le symbolisme idéologique}

\subsection{La vision ville/campagne}

La carence descriptive pourrait s'interpréter selon le présupposé suivant: les lecteurs connaissent préalablement les espaces urbains, ce sont des lieux qui leur sont familiers pour lesquels des descriptions précises ne sont pas nécessaires à l'identification et à la reconnaissance.

Ainsi s'expliquerait la différence qu'il existe entre l'espace urbain et l'espace rural, non seulement en ce qui concerne les techniques descriptives mises en œuvre mais aussi les valeurs qui leur sont associées. En effet, si l'espace urbain représente la sociabilité, la campagne sert souvent les valeurs contraires, sans être forcément un refuge agréable ou un ornement :

Lettre 108: "Cela fait, comme tu le sais, quelque temps que, fatigué par les déplaisirs que procurent un état, qu'il soit civil ou militaire, je demandai et j'obtins une mission pour les Sierras d'Alcaraz. Je partis content, emportant des poètes anciens et modernes [...] persuadé qu'en laissant la société j'échapperais aux tracas et aux tourments qui m'avaient tant fait souffrir [...]. J'ai fini alors par me connaître, car au bout de peu de temps, je me rendis compte que toutes mes inclinations avaient disparu avec moi dans ces solitudes et, me retrouvant au milieu de femmes grossières et d'hommes, bien qu'un peu plus cultivés, ne possédant que peu de recours pour la conversation, je me considérai comme Ovide, exilé du monde et j'en vins presque à haïr toute étude. En deux mots, pour te peindre mon chagrin quotidien, je te dirai que je passais mon temps à compter les heures, comme celui qui est prisonnier ou malade ${ }^{14} »$.

Cette vision de l'espace rural coïncide avec celle des personnages de la campagne qui dans l'ensemble du roman apparaissent comme des êtres grossiers, sans éducation, ce qui pose le problème du code de valeurs conditionnant la représentation de l'espace romanesque.

\subsection{Un code social et moral}

D'abord, il est évident que la vision de la campagne diverge assez de celle qui apparaitt dans le thème traditionnel de la alabanza de aldea (éloge du village ou de la vie aux champs), qui était cependant souvent utilisé dans la littérature de l'époque ${ }^{15}$. La thèse sur le monde rural qu'offre la Serafina, semble donc assez éloignée des idées de Rousseau, très en vogue à cette époque, et qui considéraient le campagnard comme "vertueux et innocent». Nous retrouvons plutôt ici les théories de La Mettrie selon lesquelles «Les hommes sont nés méchants, sans l'éducation il y en aurait peu de bons $»^{16}$. L'opposition entre ville et campagne retranscrit les idées des Lumières sur la valeur de l'éducation.

Mais en plus des idées philosophiques, l'espace sert les intentions moralisantes. En effet, comme de nombreux autres romans de cette période, les lieux de sociabilité sont utilisés comme des microcosmes de la société ${ }^{17}$. A partir de ces endroits, le narrateur principal peut, en tant que spectateur-acteur, émettre des jugements sur les 
comportements sociaux et humains de son époque. La réflexion se concentre en général autour du thème du mariage et de la famille (lettres 52, 114, 120, etc.).

Toujours dans une perspective moralisante, nous pouvons noter que certaines descriptions de lieux mettent en œuvre un symbolisme assez transparent :

Lettre 51 : « Après mille tours et détours, nous arrivâmes au bout d'une impasse, et poussant une porte étroite et branlante, nous vîmes apparaître un petit homme en haillons, contrefait et bossu. Après un escalier de pigeonnier ou de clocher et un couloir crasseux et plein de toile d'araignées, nous finîmes par arriver dans une salle délabrée, où i y avait une table couverte de feutre vert (trouée par les mégots des cigares dont la fumée dense et nauséabonde altérait la lumière et coupait la respiration) $[. . .]^{18} \%$.

Dans cet exemple, l'espace répète le jugement moral du narrateur sur le jeu et la prostitution. Les personnages de moralité douteuse ne peuvent qu'évoluer dans des endroits dégradés. En dernier ressort, la construction de l'espace dans la Serafina renvoie aux intentions analytiques et morales, inséparables de la littérature épistolaire et du roman de la Ilustración ${ }^{19}$.

\section{Conclusion}

L'espace dans le roman est bien plus qu'un simple décor. Il assume en même temps des fonctions narratives et il est inséparable de la création romanesque. De plus, l'espace littéraire doit aussi être considéré d'un point de vue diachronique : l'analyse de son évolution, de son mode de création et de représentation ouvre d'intéressantes perspectives pour l'étude historique et sociale de la littérature.

Dans ce sens, le cas de la Serafina permet de rendre compte d'une évolution formelle : la littérature du XVIII ${ }^{e}$ utilise des techniques traditionnelles (l'ornement, le tableau) mais combinées avec d'autres qui annoncent déjà le réalisme (l'utilisation systématique de toponymes, la fonctionnalité de l'espace, sa relation métonymique avec les personnages). Du point de vue de l'histoire de la littérature, c'est la preuve du changement des préceptes classiques de la représentation de la réalité, en Espagne, les écrivains de la fin du XVIII ${ }^{e}$ "se démarquent de l'imitation de la nature pour se centrer sur l'imitation des choses particulières; ils substituent de plus la nature par la société ${ }^{20} »$.

37 Sur le plan idéologique, cette façon de créer l'espace correspond avec certains présupposés de la Ilustración auxquels on pourrait donner le nom de réalisme moral : il s'agit d'illustrer par l'exemple, un exemple qui doit ressembler à la réalité afin d'être crédible.

\section{BIBLIOGRAPHIE}

AGUILAR PIÑAL Francisco, Bibliografía fundamental de la literatura española, siglo XVIII, Madrid, Sociedad General Española de Librería, 1976. 
ALVÁREZ BARRIENTOS Joaquín, La novela del siglo XVIII, Madrid, Júcar, 1991.

AUERBACH Erich, Mimesis, la représentation de la réalité dans la littérature occidentale, Paris, éd. Gallimard, 1968.

BAL Mieke, Narratologie, Utrecht, Hes Publishers, 1984.

BARTHES Roland ; BERSANI Léo ; HAMON Philippe ; RIFFATERRE Michel ; WATT, Ian, Littérature et réalité, Paris, éd. du Seuil, 1982.

CASALDUERO Joaquín, « Las nuevas ideas económicas sobre la agricultura en el siglo XVIII y el nuevo sentimiento de naturaleza », Estudios de literatura española, pp. 172-185, Madrid, Gredos, 1973.

FERRERAS Juan Ignacio, Los orígenes de la novela decimonónica (1800-1830), Madrid, Taurus, 1973.

GENETTE G., H.R. JAUSS J. M. SCHAEFFER R. SCHOLES W.D STEMPEL K. VIETOR, Théorie des genres, Paris, éd. Seuil, 1986.

GENETTE Gérard, Figures II, Paris, éd. du Seuil, 1969, 294 p., Coll. Points.

MARTI Marc, Ville et campagne dans l'Espagne des Lumières, (1746-1808), Saint Étienne, Cahiers du GRIAS, 1997.

MITTERAND Henri, Le discours du roman, Paris, PUF, 1980.

SOUBEYROUX Jacques, (dir.), Lieux dits, Recherches sur l'espace dans les textes ibériques, Cahiers du GRIAS nl, Saint Étienne, 1993.

ZIMA PIERRE V., Manuel de sociocritique, Paris, éd. Picard, 1985.

\section{NOTES}

1. Mor de Fuentes, José, La Serafina, edición, prólogo y notas de Manuel Gil Ildefonso, Caesaraugustana II, Zaragoza, 1959, 203 p.

2. A propos du roman domestique, voir Laurent Versini, Le roman épistolaire, Paris, PUF, 1979, $\mathrm{p}$ 53. Cette désignation est appliquée en particulier aux romans de Richardson et Leontine de l'allemand Léon Kotzebue, publié en 1808.

3. Jacques Soubeyroux, «Le discours du roman sur l'espace, approche méthodologique ", Lieux dits, Recherches sur l'espace dans les textes ibériques, Cahiers du GRIAS nl, Saint Étienne, 1993, p 13.

4. Gérard Genette, Figures II, Paris, éd. du Seuil, 1969, p 44.

5. Laurent Versini, Op. cit., p. 50 sq.

6. Roland Barthes, «L'effet de réel », Littérature et réalité, Paris, Seuil, 1981, pp 81-90.

7. Quant à la fonction métonymique de l'espace, voir Mieke Bal, Narratologie, Utrecht, 1983, p 108.

8. Sur le postulat de sociabilité comme fondement du roman épistolaire européen, voir Laurent Versini, Op. cit., p. 210.

9. «Ayer mañana, iba yo por Santa Engracia todo empapado en mis cavilaciones, cuando vi a una señora sentada, que me miró con notable particularidad ; reparé en ello y acercándome conocí, no sin trabajo, a la Narcisa, que de consumida y desfigurada no acababa de creer fuese la misma.

Apenas le saludé, le pedí con instancia me manifestase sus dolencias, con todas las circunstancias de su actual situación, y me habló en estos términos : [...]» carta 46 : «El otro día me encontré en la calle con la criada que acompañaba a Narcisa aquella mañana, y habiéndole preguntado por su ama, me dijo que [...]» 
10. «Ayer di la vuelta de mi expedición solitaria y aquijotada ».

11. Carta 85 : «Amigo, si yo fuese astrónomo y no estuviese enamorado, tendría un paraíso en este observatorio.

Un cielo despejadísimo es la brillante bóveda de la inmensa perspectiva que me rodea. Al norte descuella el majestuoso Pirineo, de cuyo seno se precipita el cristalino Gállego, que viene regando por largas leguas esta huerta fertilísima, compuesta por la mayor parte de frondosos viñedos. Al poniente, por una parte se tiende la vista sobre la apacible corriente del Ebro y los llanos de la Bardena, hasta internarse en el corazón de Navarra

Y por la otra el Moncayo entredorado

Campea con la ráfaga lumbrosa

De aquel celaje que en su sien reposa

pues en la estación actual no tiene coronada de nieve la alta frente, como dice mi paisano Argensola. Casi al mediodía se descubre perfectamente Zaragoza, situada en un llano, con sus torres empinadas, que la hacen mucho más vistosa desde fuera que Valencia, Barcelona y otras ciudades principales. A su espalda asoma el antiguo sequeral de Torrero, transformado en el vergel más delicioso por los desvelos del inmortal Pignatelli. Más a la izquierda, se vuelve a aparecer el Ebro, y luego a levante lo desvían, y a trechos lo encajonan, las sierras inmediatas que son, por lo más, bastante estériles, y con su triste aspecto forman el claroscuro de este incomparable cuadro ».

12. Sur le Rococo en littérature voir José M. Caso González, "Cadalso y la poética rococó », Coloquio internacional sobre José Cadalso, Abano Terme, Piovane ed., 1985, pp. 49-62: «Dans la structure rococo, sans perdre l'unité d'ensemble, les éléments constitutifs sont conçus comme des éléments isolés et isolables, que l'on peut contempler pour eux-mêmes. [...] les ornements ont une valeur indépendante [...] Cependant, il me semble que l'on ne peut pas parler d'un style rococo stricto sensu, mais plutôt de caractères (sociaux, culturels, littéraires) qui conditionnent une certaine façon de faire de la littérature ».

«En la estructura rococó, sin perderse la unidad del conjunto, sus componentes se conciben como elementos aislados y aislables, capaces de ser contemplados en sí mismos [...] Los adornos tienen valor independiente [...] Sin embargo, no me parece que se pueda hablar de un estilo rococó en términos estrictos, pero sí de unos caracteres (sociales, culturales, literarios) que conforman una determinada manera de hacer literatura ».

13. Sur la nouvelle vision de la nature, voir Joaquín Casalduero, «Las nuevas ideas económicas sobre la agricultura en el siglo XVIII y el nuevo sentimiento de naturaleza », Estudios de literatura española, pp. 172-185, Madrid, Gredos, 1973.

14. Carta 108 : « Hace, como sabes, algún tiempo, que acongojado con los desabrimientos que trae consigo un cuerpo, sea militar, sea civil, solicité y conseguí una comisión para las Sierras de Alcaraz. Fuime gozosísimo, pertrechado de poetas antiguos y modernos [...] persuadido de que al dejar la sociedad, me desprendería de los anhelos y zozobras que tanto me habían atormentado [...]. Entonces me acabé de conocer, pues a poco tiempo advertí que todas mis inclinaciones se habían emboscado conmigo por aquellas soledades, y al verme en medio de unas mujeres tosquísimas y de unos hombres, aunque más regulares, de pocos recursos para la conversación, me consideré, como Ovidio, desterrado del mundo y casi aborrecí de todo punto el estudio. Para pintarte en dos palabras mi habitual desconsuelo te diré que pasaba el tiempo en contar las horas, como quien está preso o enfermo [...]».

15. Voir notre travail, Ville et campagne dans l'Espagne des Lumières (1746-1808), Saint Étienne, Publications de l'Université de Saint Étienne, Cahiers du GRIAS, 1997.

16. Cité par Adam Antoine, Le mouvement philosophique dans la première moitié du XVIIIe siècle, Paris, Société d'Édition de l'Enseignement Supérieur de la Sorbonne, 1967, p. 108.

17. Une technique utilisé dans les Cartas marruecas de José Cadalso.

18. Carta 51 : « Después de mil vueltas y revueltas, llegamos al extremo de un callejón sin salida, y empujando una puerta estrecha y desquiciada, se presentó un hombrezuelo andrajoso, 
contrahecho y derrengado [...] Tras una escalera de palomar o de campanario y un corredor sucio y telarañiento, vinimos a parar en un sala desmantelada, donde había una mesa cubierta de bayeta verde (acribillada con las pavesas de los cigarros, cuyo humo denso y hediondo ofuscaba las luces y privaba la respiración) [...]»

19. Laurent Versini, Op. cit., p. 56-57.

20. Alvárez Barrientos, Joaquín, La novela del siglo XVIII, Madrid, Júcar, 1991, p. 396.

\section{AUTEUR}

MARC MARTI

Université de Nice-Sophia Antipolis 